\title{
O Inquérito de soroprevalência da infecção chagásica humana (1975-1980)
}

\author{
Seroprevalence survey of human Chagas' infection (1975-1980)
}

\author{
Antônio Carlos Silveira*, Guilherme Rodrigues da Silva† e Aluízio Prata**
}

\begin{abstract}
RESUMO
O Inquérito sorológico da prevalência de infecção chagásica no Brasil, 1975/1980, cujos resultados foram objeto de publicação anterior, em 1984 (Camargo e cols), é aqui detalhado em sua distribuição geográfica. Foi uma iniciativa da Superintendência de Campanhas de Saúde Pública (SUCAM/MS) e do Conselho Nacional de Pesquisas (CNPq) através de seu Programa Integrado de Doenças Endêmicas (PIDE), tendo servido para delimitar e estratificar mais precisamente a área com transmissão endêmica da doença de Chagas e orientar as ações de controle vetorial, implementadas a partir da segunda metade da década de 1970. Mostrou uma soroprevalência estimada de 4,22\% da infecção chagásica para a população geral residente em área rural no país. Observe-se que não foram incluídos o Estado de São Paulo e o Distrito Federal, para onde se acreditava já haver informação suficiente e recente. Abrangeu todas as demais unidades federativas, com o exame de 1.626 .745 amostras de sangue, processadas por imunofluorescencia indireta. Destas, foram consideradas válidas para efeito de processamento e análise estatística 1.352.197, procedentes de 3.026 municípios de 24 estados, segundo a divisão política de então. Os resultados no geral foram confirmatórios em relação ao que era já conhecido. Alguns achados no entanto não corresponderam ao esperado, o que foi objeto de investigação ou se soube depois justificáveis, com base em dados de entomologia e outros que serviram à interpretação dos resultados.
\end{abstract}

Palavras-chaves: Doença de Chagas. Inquérito sorológico. Soroprevalência. Brasil.

\begin{abstract}
The results of the Serological survey for the prevalence of chagasic infection in Brazil, 1975/1980, were published before (Camargo et al, 1984) but the detailed geographical distribution is described in this paper. This was an initiative of two governmental bodies: the Superintendencia de Campanhas de Saúde Pública a Ministry of Health Division in charge of all public health policies, and, the Research National Council of the Ministry of Science and Technology through a program called Integrated Program for Endemic Diseases. Results obtained were extremely useful for a precise delimitation of the area with endemic transmission of Chagas disease, and hence, to direct the activities for vector control, which were implemented from 1975 onwards. This survey showed an estimated seroprevalence of $4.2 \%$ of $T$. cruzi infection in the rural population in the country. Only two federal units were not included, São Paulo and the Federal District (Brasilia) for which enough recent information was available. This survey included all the other federal units of Brazil, with the examination of 1,626,745 blood samples by indirect immunofluorescence. From them, 1,352,197 were validated for processing and statistical analysis, which were from 3,026 municipalities of 24 states, as by the political division of the country by this time. Overall results obtained, confirmed data that were well known, but some were non expected. These last were subject of further investigations, until confirmation, based also on entomological data and a better interpretation of the results obtained.
\end{abstract}

Key-words: Chagas disease. Serological survey. Seroprevalence. Brazil.

Ainda que os resultados do inquérito de soroprevalência nacional tenham sido já publicados ${ }^{1}$, duas são as razões para que sejam aqui novamente reportados. A primeira delas está no escopo do próprio documento que se está produzindo. Sem o presente artigo a História do Controle da Doença de Chagas no Brasil estaria incompleta. A segunda razão deve-se ao fato de que, revisando o banco de dados disponível junto ao Departamento de Medicina Preventiva da Universidade de São Paulo, aos cuidados de um dos autores do artigo original (Guilherme Rodrigues da Silva), julgou-se pertinente fazer a divulgação de informações mais detalhadas do que aquelas antes veiculadas, como a distribuição da proporção de sororreagentes por município, afora outras, de caráter complementar.

\footnotetext{
*Ex-Diretor da Divisão Nacional de Doença de Chagas e da Divisão Nacional de Epidemiologia do Ministério da Saúde e Consultor da Organização Panamericana de Saúde, Brasília, DF. ${ }^{* *}$ Doutor em Medicina Tropical, Universidade Federal do Triângulo Mineiro, Uberaba, MG. †In memoriam. Endereço para correspondência: Dr. Antônio Carlos Silveira. SQN 304/B/302, 70736-020 Brasília, DF.

e-mail: atcrs@uol.com.br
}

\section{INTRODUÇÃO: ANTECEDENTES E OS PROPÓSITOS DO ESTUDO}

Na segunda metade dos anos 70, diante da perspectiva de se regularizar as ações de controle da doença de Chagas, com o aporte adicional de recursos que deveria ser proporcionado pela desativação de atividades do programa de malária em áreas com transmissão interrompida, haveria que atualizar o conhecimento não só sobre a distribuição dos vetores, foco principal das atividades a serem implementadas, como também sobre a prevalência da infecção humana no país. Em função disso, a Superintendência de Campanhas de Saúde Pública (SUCAM) do Ministério da Saúde, em associação com o Conselho Nacional de Pesquisas (CNPq), através do recém criado Programa Integrado de Doenças Endêmicas (PIDE), promoveu estudo sorológico com o propósito de estimar a prevalência da infecção chagásica na população geral residente em área rural.

O inquérito, que se realizou entre 1975 e 1980, foi o primeiro estudo sobre a infecção chagásica com alcance nacional. Antes disso, ainda que houvesse muitos dados sobre a soroprevalência da doença no país, a informação era fragmentada, produto de estudos diversos em diferentes áreas e que seguiram diferentes 
métodos e técnicas de colheita e processamento laboratorial. Isso fazia com que os resultados fossem pouco comparáveis.

$\mathrm{Na}$ tabela abaixo, procurou-se reunir alguns dos dados até aquela ocasião disponíveis, ao menos aqueles mais significativos do ponto de vista histórico e com informações mais relevantes em relação à situação epidemiológica da doença de Chagas ${ }^{1-12}$ (Tabela 1).

Mais ou menos extensos, alguns desses estudos compreenderam áreas ou populações selecionadas e, outras vezes, foram de caráter exploratório no sentido de determinar a ocorrência ou não da enfermidade. As diferenças observadas nos resultados em alguma medida dependeram desse diferente tipo de abordagem e dos objetivos em cada caso pretendidos.

Vale observar que em alguns Estados, como Espírito Santo ${ }^{13}$, Rio de Janeiro ${ }^{14}$ e Santa Catarina ${ }^{15}$, foram também realizados inquéritos sorológicos, que mostraram ser a doença de Chagas inexistente ou um problema de menor importância em termos de saúde pública. Entre estes, apenas no Rio de Janeiro se comprovou a presença de Triatoma infestans, principal vetor da enfermidade no país, em foco circunscrito a apenas dois municípios ${ }^{16}$.

Para outros Estados, como Piauí, Maranhão, Mato Grosso e Mato Grosso do Sul, além daqueles da Amazônia, o conhecimento existente era muito escasso, havendo não mais do que dados entomológicos esparsos e o registro de alguns poucos casos de infecção.

Assim, pelos dados aqui coligidos, se pode melhor avaliar a necessidade que havia em se fazer naquela ocasião um inquérito nacional de soroprevalência, que servisse para atualizar e complementar a informação sobre a distribuição da endemia chagásica, precisar a área que deveria ser objeto de controle e definir quais aquelas a serem prioritariamente cobertas.

\section{MÉTODOS}

\section{Amostragem}

A população alvo foi aquela residente na área rural brasileira. A principio todos os municípios existentes deveriam ser amostrados, com exceção daqueles do Estado de São Paulo e do Distrito Federal, para onde se considerava haver já informação suficiente e recente. Localidades rurais constituídas por mais de 500 casas foram também excluídas da amostra, assumindo-se que as mesmas teriam, na maioria das vezes, estrutura e organização urbanas. Com isso, alguns poucos municípios não atenderam os critérios de inclusão, por não apresentarem localidades caracteristicamente rurais. Por outra parte, localidades com 10 ou menos casas foram também excluídas, pelas dificuldades operacionais que representava sua inclusão e pelo fato de que um pequeno número de casas proporcionaria um limitado número de amostras de sangue colhidas. Apenas para a Amazônia o inquérito abarcou essas localidades com um número menor de habitações, em função de sua alta frequência na região.

Para cada município as localidades foram agrupadas em cinco diferentes estratos: localidades de 10 a 30 casas; de 31 a 50; de 51 a 100; de 101 a 200; e de 201 a 500. Para cada estrato foi aplicado um diferente percentual de localidades a amostrar, de $10 \%$ para os dois primeiros estratos; e de 15,20 e $25 \%$ para os seguintes. As localidades foram selecionadas aleatoriamente. Selecionadas as localidades, o número de casas a visitar em cada estrato foi definido com base naqueles mesmos percentuais aplicados agora de forma invertida, ou seja, de $25 \%$ para o estrato de 10 a 30 casas; de $20 \%$ para aquele de 50 a 100 casas; de $15 \%$ para o de 101 a 200 casas; e $10 \%$ para os dois outros (de 10 a 30 e de 31 a 50 casas). As casas foram também selecionadas de forma aleatória, por sorteio. Fez-se a coleta de sangue de todos os moradores das casas selecionadas.

\section{Coleta das amostras de sangue}

Procurou-se padronizar o mais possível os procedimentos no sentido de garantir uniformidade das condições para exame, evitando assim qualquer possível distorção nos resultados.

Na perspectiva de que se fosse alcançar mais de um milhão de amostras, o que de fato se confirmou, optou-se por fazer a colheita de material em papel filtro. As amostras de sangue foram tomadas por punção, com lanceta descartável, da polpa digital do dedo anelar da mão esquerda e fazendo-se sua impregnação em tiras de papel filtro de 12,5 x 5cm. Cada uma delas com espaço suficiente para conter 8 amostras, que reunidas em cadernos de 5 folhas, intercaladas por papel impermeável, formavam pequenos blocos que permitiam a coleta de até 20 amostras em duplicata.

O papel de filtro utilizado (Klabin $80 \AA$ ) foi especialmente desenvolvido para o inquérito, de forma controlada, isento de impurezas e previamente testado.

TABELA 1 - Alguns inquéritos de soro-prevalência da infecção chagásica, anteriores ao Inquérito Sorológico Nacional (Brasil, 1975-1980).

\begin{tabular}{|c|c|c|c|}
\hline Autor(es) & Ano & Area de abrangência & $\begin{array}{c}\text { Resultados } \\
\text { (proporção de soropositivos/100) }\end{array}$ \\
\hline Villela $^{2}$ & 1930 & Belo Horizonte/MG & 28,4 \\
\hline Pedreira de Freitas ${ }^{3}$ & 1947 & Municípios de SP e MG & 51,3 \\
\hline Dias, Laranja \& Pellegrino ${ }^{4}$ & 1948 & Bambuí/MG & 38,4 \\
\hline Freitas \& Figueiredo 5 & 1951 & Hidrolândia e Trindade/GO & 13,3 e 27,5 \\
\hline Brant cols 6 & 1957 & Municípios endêmicos (4) do RS & 23,9 \\
\hline Pinotti (apud Martins AV) ${ }^{7}$ & 1958 & Municípios endêmicos (4) do PR & 13,9 \\
\hline Ponde 8 & 1960 & Salvador/BA & 25,0 \\
\hline Lucena $^{9}$ & $1957-1962$ & Municípios de AL, PB, PE, RN & AL: 19,1; PE: 17,0 ;PB: 20,7 ; RN: 12,2. \\
\hline Porto \& Porto 10 & 1962 & Triângulo Mineiro/MG & 47,3 \\
\hline Salgado \& Pellegrino ${ }^{11}$ & 1963 & Municípios (82) de MG & 6,2 \\
\hline Alencar cols ${ }^{12}$ & 1963 & Regiões do Cariri e Baturité/CE & 22,6 \\
\hline Silva (apud Camargo cols) ${ }^{1}$ & 1964 & Municípios (285) de SP & 13,9 \\
\hline
\end{tabular}


Feita a colheita o doador era identificado atribuindo-se a ele, um número sequencial para cada localidade, e que era apontado junto à amostra no próprio papel filtro e em formulário (Boletim Diário de Coleta de Sangue) destinado ao registro de dados referentes a estado, município, localidade de origem, e dos códigos de identificação a eles previamente estabelecidos. Aí se fazia também o registro de idade, sexo e local de nascimento de cada participante da amostra, e dos resultados dos exames pelo laboratório de referência.

Concluído o trabalho em determinada localidade, o material era embalado, sendo o(s) bloco(s) com as amostras envoltos no(s) formulário(s) correspondentes e presos com elástico, colocado em saco plástico e armazenado em caixa de isopor, para proteção contra umidade e eventuais grandes variações de temperatura. Semanalmente, se fazia o transporte das caixas contendo a produção daquele período às sedes regionais da SUCAM, onde amostras e formulários eram conferidos e a seguir enviados para o laboratório de referência. Até seu processamento, eram conservadas a $-20^{\circ} \mathrm{C}$. As duplicatas de amostras já processadas se remetia ao laboratório central, para controle de qualidade.

Processamento das amostras de sangue e controle de qualidade

O teste de escolha para o processamento das amostras foi a imunofluorescência indireta, em função de sua simplicidade técnica, baixo custo e facilidade de padronização ${ }^{1,17}$.

Contribuiu também para a escolha deste teste o fato de que vários laboratórios existentes no país, de instituições de ensino, pesquisa e de saúde pública, estavam capacitados para sua realização. Isso permitiu a constituição de uma rede de 14 laboratórios regionais, localizados em diferentes estados, que serviram de referencia para os exames de material colhido naquelas áreas de sua influencia ou cobertura, previamente definidas. Ainda assim houve ocasiões em que foi necessária a transferência de amostras de um a outro laboratório.

Todo o trabalho de processamento das amostras de sangue esteve sob coordenação do Laboratório de Imunologia e Soroepidemiologia do Instituto de Medicina Tropical de São Paulo, que desempenhou as funções de laboratório central para o inquérito. A ele coube o treinamento de pessoal, o suprimento de insumos, a preparação e distribuição de reagentes e o permanente controle de qualidade dos testes realizados pelos laboratórios regionais.

Para o controle de qualidade, de cada lote de amostras processadas eram escolhidas ao azar de 10 a $20 \%$ das duplicatas recebidas, para serem submetidas a novos testes pelo laboratório central. o controle se fazia pela determinação de índices de concordância, co-positividade e co-negatividade. Quando necessário, se buscou determinar as eventuais causas de discordância e sua correção.

\section{Processamento e análise de dados}

Os dados registrados no Boletim Diário de Colheita de Sangue, logo que validados pela checagem manual de sua correspondência com as amostras recebidas e verificada sua origem (estado, município, localidade e seus códigos), eram digitados para fitas magnéticas, que passaram a compor o banco de dados do inquérito. Estes arquivos passavam a seguir, já com o uso de recursos de processamento eletrônico, por uma fase de crítica ou de avaliação de sua consistência. E, tal como informado no trabalho anteriormente publicado "foram feitas as seguintes tabulações para cada unidade federativa: distribuição dos dados amostrais por localidades de cada município segundo autoctonia, grupo etário, sexo e por contagem de indivíduos positivos; distribuição das observações amostrais por estrato de cada município segundo autoctonia, grupo etário, sexo e porcentagem de reações positivas; distribuição amostral para cada localidade de cada município em termos de frequências relativas de reações positivas segundo autoctonia, grupo etário e sexo; distribuição das observações amostrais segundo as mesmas variáveis anteriores para cada estrato de cada município; e, finalmente, a distribuição por município e por seus estratos segundo a fração amostral, número esperado de positivos, tamanho da população e a estimativa de prevalência de reações sorológicas positivas"1.

Importa assinalar que no cálculo das estimativas de prevalência todas as reações com resultados duvidosos foram consideradas como negativas. 0 detalhamento dos critérios seguidos para as estimativas de prevalência foram objeto de trabalho apresentado na I Reunião Anual de Pesquisa Aplicada sobre Doença de Chagas ${ }^{18}$.

\section{RESULTADOS E DISCUSSÃO}

Foram ao todo examinadas 1.626 .745 amostras de sangue, das quais foram consideradas válidas ou úteis para efeito de processamento e análise estatística 1.352.197, procedentes de 3.026 municípios de 24 estados, segundo a divisão política de então e considerando que São Paulo e Distrito Federal não participaram do estudo.

Nas Figura 1 e Tabela 2 mostra-se as estimativas de prevalência por estado e para o país. É de se destacar aí que: I) em muitos casos os resultados foram confirmatórios em relação ao já conhecido, como as altas taxas de soro-prevalência para o Rio Grande do Sul, Minas Gerais, Bahia e Goiás; II) assim foi também para estados em que se esperava uma baixa ou muito baixa sororreatividade, como para a maior parte dos estados da Amazônia, Espírito Santo e Santa Catarina; III) outras vezes estiveram aquém do que se estimava, com base em resultados de inquéritos anteriores, como aconteceu com o Ceará; IV) ao contrário, para Estados como Piauí, Mato Grosso, Acre e Amazonas as estimativas de prevalência superaram muito o que era esperado (Figura 1 e Tabela 2).

A elucidação dos achados que não correspondiam ao que indicavam, ou poderiam sugerir, os dados até então existentes, tiveram diferente desdobramento. Algumas situações foram objeto de investigação, enquanto em outros casos se encontrou explicação em informações complementares. Para o Mato Grosso e Piauí, a informação entomológica dos levantamentos de base mostraram a presença, antes disso desconhecida, de Triatoma infestans naqueles estados ${ }^{19}$. Além disso, para o Piauí se verificou grandes infestações, com colonização intradomiciliar, de Triatoma brasiliensis. 


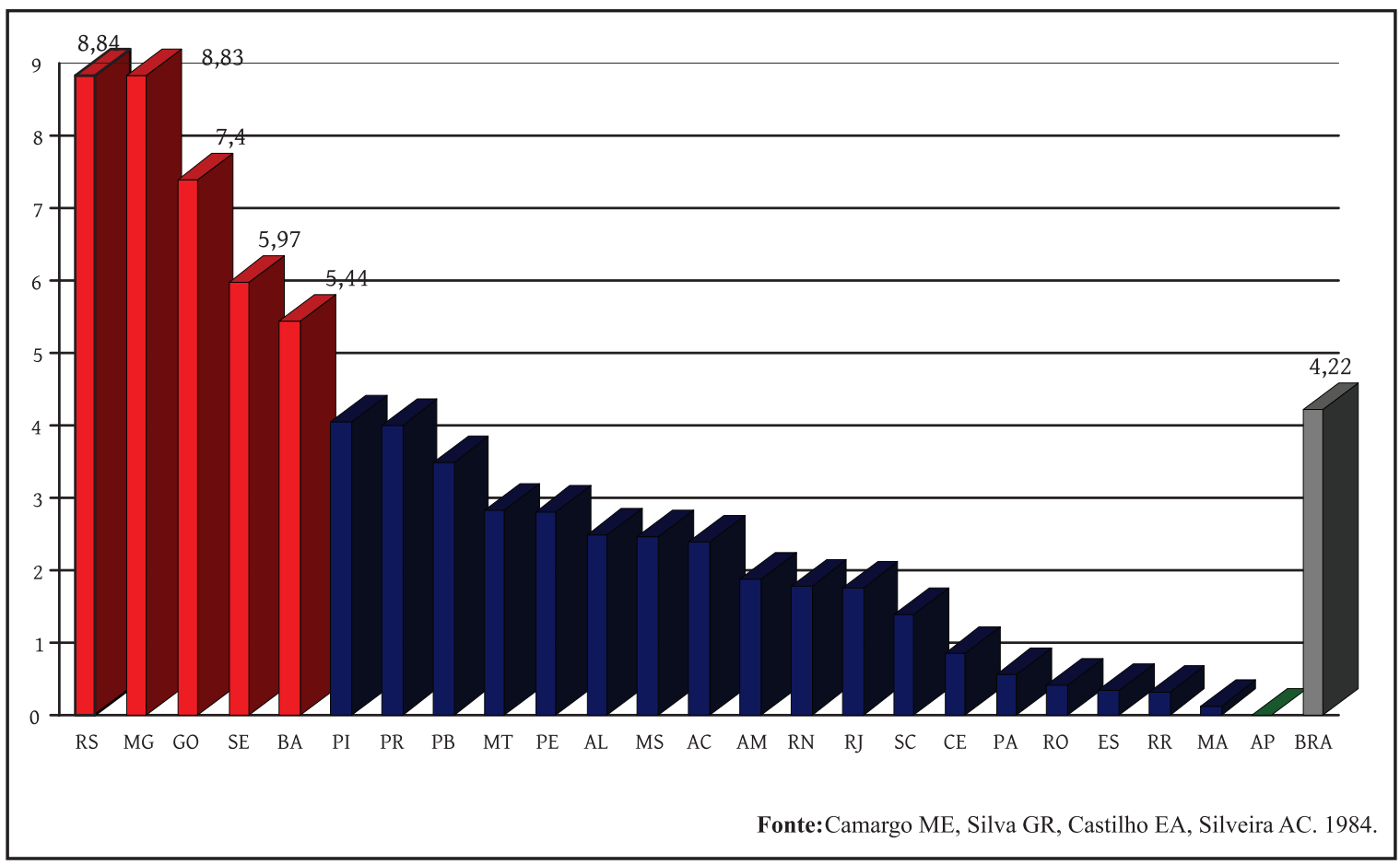

FIGURA 1 - Soroprevalência da infecção chagásica. Distribuição por estado. Inquérito sorológico nacional. Brasil. 1975-1980.

TABELA 2 - Estimativa de prevalencia por estado e país no inquérito sorológico de prevalência da infecção chagásica no Brasil, 1975-1980.

\begin{tabular}{lc}
\hline Estado & $\begin{array}{c}\text { Estimativa de prevalência } \\
\text { (por 100) }\end{array}$ \\
\hline Rio Grande do Sul & 8,84 \\
Minas Gerais & 8,83 \\
Goiás & 7,40 \\
Sergipe & 5,97 \\
Bahia & 5,44 \\
Piauí & 4,04 \\
Paraná & 4,00 \\
Paraíba & 3,48 \\
Mato Grosso & 2,82 \\
Pernambuco & 2,79 \\
Alagoas & 2,48 \\
Mato Grosso do Sul & 2,46 \\
Acre & 2,39 \\
Amazonas & 1,88 \\
Rio Grande do norte & 1,78 \\
Rio de Janeiro & 1,75 \\
Santa Catarina & 1,39 \\
Ceará & 0,84 \\
Pará & 0,56 \\
Rondônia & 0,41 \\
Espírito Santo & 0,32 \\
Roraima & 0,31 \\
Maranhão & 0,12 \\
Amapá & 0,00 \\
BRASIL & 4,22 \\
\hline Fonte: Camis & \\
\hline
\end{tabular}

Fonte: Camargo ME, Silva GR, Castilho EA, Silveira AC. 1984.
Em relação ao Ceará, as diferenças observadas entre os resultados se considerou que poderia ser atribuída à diferente abrangência dos estudos realizados.

No Estado do Amazonas, foram identificados municípios do alto Rio Negro com elevadas taxas de soroprevalência (Barcelos, Novo Airão e Japurá), superiores inclusive à média nacional. Investigação aí realizada aventou a hipótese de transmissão extradomiciliar por Rhodnius brethesi, associada à extração de piaçaba ${ }^{20}$, o que foi confirmado em trabalhos posteriores na área ${ }^{21}$. Uma outra situação na Amazônia que mereceria ter sido melhor estudada foi aquela do Estado do Acre, para onde se aceitou, sem que se houvesse efetivamente demonstrado, que os altos índices de soropositividade seriam devidos à migração desde áreas endêmicas.

Outro dado mais agregado, que se exibe a seguir, se refere à distribuição dos resultados por grupo de idade (Figura 2), que aqui foram arbitradas em função do interesse em se mostrar: I) que os resultados obedeceram ao perfil conhecido de distribuição etária para a doença de Chagas, em função de sua história natural; II) e, que havia na ocasião importante transmissão ativa, a julgar pela proporção de sororreagentes no grupo de menores de 4 anos e mesmo naquele de 5 a 14 anos de idade (que corresponderam, somados, a aproximadamente 47\% da população amostrada) (Figura 2).

Em relação à distribuição da soropositividade por área, de acordo com a malha municipal do país pelo censo do Instituto Brasileiro de Geografia e Estatística (IBGE), de $1980^{22}$, um primeiro dado a fazer referência é que do total de 3.026 municípios investigados em apenas 367 (12,1\%) deles não houve sororreagentes. A maior parte deles (em termos relativos ao total de unidades municipais existentes) 


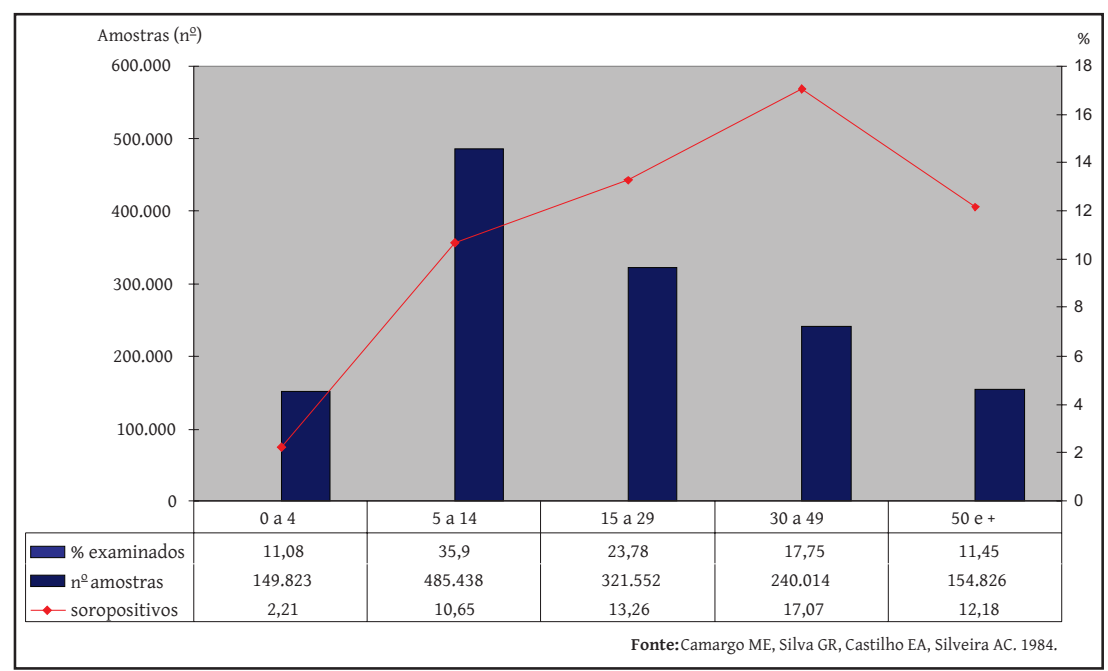

FIGURA 2 - Número e percentual de amostras examinadas e proporção de soropositivos por grupo de idade. Inquérito sorológico nacional. Brasil. 1975-1980.

TABELA 3A - Número de municípios e população residente ${ }^{1}$ segundo intervalos de classe de soro-positividade no inquérito sorológico nacional no estado do Rio Grande do Sul, 1975-1979.

\begin{tabular}{lcccc}
\hline Soro-positividade & № Municípios & \% & № Habitantes & $\%$ \\
\hline 0,0 & 42 & 18,1 & 531.151 & 6,8 \\
$0,1-2,0$ & 59 & 25,4 & 1.820 .839 & 23,4 \\
$2,1-5,0$ & 34 & 14,7 & 2.038 .785 & 26,2 \\
$5,1-10,0$ & 32 & 13,8 & 1.374 .004 & 17,7 \\
$10,1-30,0$ & 50 & 21,6 & 1.663 .805 & 21,4 \\
$30,1-50,0$ & 13 & 5,6 & 317.620 & 4,1 \\
$\geq 50,1$ & 1 & 0,4 & 14.366 & 0,2 \\
IGN & 1 & 0,4 & 16.642 & 0,2 \\
\hline Total & $\mathbf{2 3 2}$ & $\mathbf{1 0 0}$ & $\mathbf{7 . 7 7 7 . 2 1 2}$ & $\mathbf{1 0 0}$ \\
\hline
\end{tabular}

${ }^{1}$ censo IBGE $1980,{ }^{2}$ não incluídos na amostra.

TABELA 3B - Número de municípios e população residente ${ }^{1}$ segundo $^{2}$ intervalos de classe de soro-positividade no inquérito sorológico nacional no estado de Minas Gerais, 1975-1979.

\begin{tabular}{|c|c|c|c|c|}
\hline Soro-positividade & № Municípios & $\%$ & № Habitantes & $\%$ \\
\hline$\overline{0,0}$ & 144 & 19,9 & 2.870 .668 & 21,4 \\
\hline $0,1-2,0$ & 190 & 26,3 & 3.158 .868 & 23,6 \\
\hline $2,1-5,0$ & 85 & 11,8 & 1.917 .094 & 14,3 \\
\hline $5,1-10,0$ & 68 & 9,4 & 1.260 .035 & 9,4 \\
\hline $10,1-30,0$ & 168 & 23,3 & 3.064 .322 & 22,9 \\
\hline $30,0-50,0$ & 55 & 7,6 & 944.847 & 7,1 \\
\hline$\geq 50,1$ & 8 & 1,1 & 76.282 & 0,6 \\
\hline $\mathrm{IGN}^{2}$ & 4 & 0,6 & 93.327 & 0,7 \\
\hline Total & 722 & 100 & 13.385 .443 & 100 \\
\hline
\end{tabular}

${ }^{1}$ censo IBGE $1980,{ }^{2}$ não incluídos na amostra.

TABELA 3C - Número de municípios e população residente ${ }^{1}$ segundo intervalos de classe de soro-positividade no inquérito sorológico nacional no estado de Goiás, 1980.

\begin{tabular}{lcccc}
\hline Soro-positividade & № Municípios & \% & № Habitantes & $\%$ \\
\hline 0,0 & 9 & 4,1 & 57.409 & 1,5 \\
$0,1-2,0$ & 30 & 13,6 & 348.634 & 9,0 \\
$2,1-5,0$ & 60 & 27,1 & 957.201 & 24,8 \\
$5,1-10,0$ & 62 & 28,1 & 930.106 & 24,1 \\
$10,1-30,0$ & 58 & 26,2 & 770.738 & 19,9 \\
$30,0-50,0$ & 0 & 0,0 & 0 & 0,0 \\
$\geq 50,1$ & 0 & 0,0 & 0 & 0,0 \\
IGN & 4 & 0,9 & 801.394 & 20,7 \\
\hline Total & $\mathbf{2 2 3}$ & $\mathbf{1 0 0}$ & $\mathbf{3 . 8 6 5 . 4 8 2}$ & $\mathbf{1 0 0}$ \\
\hline
\end{tabular}

${ }^{1}$ censo IBGE $1980,{ }^{2}$ não incluídos na amostra.
TABELA 3D - Número de municípios e população residente ${ }^{1}$ segundo intervalos de classe de soro-positividade no inquérito sorológico nacional no estado de Sergipe, 1975-1980.

\begin{tabular}{lcccc}
\hline Soro-positividade & № Municípios & \% & № Habitantes & $\%$ \\
\hline 0,0 & 14 & 18,9 & 118.455 & 10,4 \\
$0,1-2,0$ & 10 & 13,5 & 174.458 & 15,3 \\
$2,1-5,0$ & 15 & 20,3 & 445.778 & 39,0 \\
$5,1-10,0$ & 18 & 24,3 & 196.101 & 17,2 \\
$10,1-30,0$ & 16 & 21,6 & 202.069 & 17,7 \\
$30,0-50,0$ & 1 & 1,4 & 4.973 & 0,4 \\
$\geq 50,1$ & 0 & 0, & 0 & 0,0 \\
IGN & 0 & 0 & 0 & 0,0 \\
\hline Total & $\mathbf{7 4}$ & $\mathbf{1 0 0}$ & $\mathbf{1 . 1 4 1 . 8 3 4}$ & $\mathbf{1 0 0}$ \\
\hline
\end{tabular}

${ }^{1}$ censo IBGE $1980,{ }^{2}$ não incluídos na amostra.

TABELA 3E - Número de municípios e população residente ${ }^{1}$ segundo intervalos de classe de soro-positividade no inquérito sorológico nacional no estado da Bahia, 1975-1978.

\begin{tabular}{lcccc}
\hline Soro-positividade & No $^{\circ}$ Municípios & \% & № Habitantes & $\%$ \\
\hline 0,0 & 30 & 8,9 & 469.242 & 4,9 \\
$0,1-2,0$ & 120 & 35,7 & 3.077 .350 & 32,5 \\
$2,1-5,0$ & 44 & 13,1 & 1.142 .965 & 12,1 \\
$5,1-10,0$ & 73 & 21,7 & 3.374 .747 & 35,6 \\
$10,1-30,0$ & 60 & 17,9 & 1.279 .989 & 13,5 \\
$30,0-50,0$ & 3 & 0,9 & 40.242 & 0,4 \\
$\geq 50,1$ & 1 & 0,3 & 16.488 & 0,2 \\
IGN ${ }^{2}$ & 5 & 1,5 & 73.240 & 0,8 \\
\hline Total & $\mathbf{3 3 6}$ & $\mathbf{1 0 0}$ & $\mathbf{9 . 4 7 4 . 2 6 3}$ & $\mathbf{1 0 0}$ \\
\hline
\end{tabular}

${ }^{1}$ censo IBGE $1980,{ }^{2}$ não incluídos na amostra.

pertencente à Amazônia. Por outro lado, em 95 municípios as taxas de soro-prevalência foram superiores a $30,1 \%$, e em 10 destes estiveram acima de 50,1\%. Nesse caso concentrados em sua quase totalidade naqueles estados com uma proporção de soropositivos maior do que os $4,2 \%$ estimados para o país (reiterase que aqui se está considerando a população geral rural brasileira, sem computar aquela correspondente ao Estado de São Paulo e ao Distrito Federal). Na Tabela 3A-E, discrimina-se o número de municípios destes estados (Rio Grande do Sul, Minas Gerais, Goiás, Sergipe e Bahia) segundo os intervalos de classe que originalmente adotou-se para categorizá-los segundo as estimativas de prevalência. 
Em que pese serem esses estados aqueles com maiores taxas de soroprevalência, note-se que mesmo aí houve um significativo número de municípios sem nenhuma amostra positiva. Isso revela que a distribuição espacial da infecção chagásica é bastante desigual, mesmo em áreas geograficamente próximas. As condições necessárias para que haja transmissão dependem de condições locais. Esse fato significa que, na medida em que os dados são discriminados e examinados em menor escala, mostram que a infecção/doença de Chagas é sempre focal, e dependente da existência ou não de determinados requisitos ou variáveis que favorecem ou não sua transmissão domiciliar. Entre elas, seguramente estão os determinantes de natureza econômica que se traduzem em precárias condições de vida e especialmente de habitação das populações sob risco.

Buscando discriminar, tanto quanto possível, as informações de soro-prevalência por município a partir do banco de dados original, e tomando-se agora a malha municipal correspondente ao Censo IBGE $1991^{23}$ e outros intervalos de classe para os resultados, fêz-se o mapeamento desses dados, conforme exibido na Tabela 4 e Figura 3.

Nem sempre foi possível estabelecer uma precisa correspondência entre os dados colhidos e a divisão política mais atual que se pode dispor. Muitas foram as novas municipalidades criadas e, algumas vezes, com território formado a partir de diferentes outros municípios.

Essa nova disposição dos dados confirmaram: I) o quão desiguais podem ser, e de fato foram, os resultados para municípios de um mesmo estado; II) que para os estados da região nordeste houve uma maior homogeneidade na sororeatividade, com exceção da Bahia, onde os índices foram mais importantes, e para o Maranhão, onde houve um grande número de municípios com nenhuma amostra positiva; III) que alguns municípios de determinadas áreas com condições ambientais e sócio-econômicas similares, registraram índices

TABELA 4 - Número de municípios por estado(1) ${ }^{(1)}$ segundo proporção de sorologia positiva no inquérito nacional de soro-prevalência. Brasil. 1975-1980.

\begin{tabular}{|c|c|c|c|c|c|c|c|}
\hline \multirow[b]{2}{*}{ Estado por Macrorregião } & \multicolumn{7}{|c|}{ Número de municípios segundo proporção de soropositivos } \\
\hline & 0 & $<4.99$ & $<9.99$ & $<19.99$ & $>20.0$ & sem informação ${ }^{(2)}$ & total \\
\hline Norte & 53 & 125 & 15 & 8 & 0 & 97 & 298 \\
\hline $\mathrm{AC}$ & 0 & 2 & 0 & 0 & 0 & 21 & 23 \\
\hline RO & 0 & 7 & 0 & 0 & 0 & 5 & 12 \\
\hline AM & 8 & 36 & 0 & 0 & 0 & 18 & 62 \\
\hline RR & 0 & 2 & 0 & 0 & 0 & 6 & 8 \\
\hline PA & 37 & 46 & 0 & 0 & 0 & 22 & 105 \\
\hline $\mathrm{AP}$ & 5 & 0 & 0 & 0 & 0 & 4 & 9 \\
\hline TO & 3 & 32 & 15 & 8 & 0 & 21 & 79 \\
\hline Nordeste & 284 & 858 & 130 & 68 & 17 & 152 & 1.509 \\
\hline MA & 78 & 51 & 0 & 0 & 0 & 7 & 136 \\
\hline PI & 11 & 74 & 15 & 14 & 0 & 4 & 118 \\
\hline $\mathrm{CE}$ & 49 & 89 & 0 & 0 & 0 & 40 & 178 \\
\hline $\mathrm{RN}$ & 39 & 102 & 7 & 0 & 0 & 4 & 152 \\
\hline PB & 20 & 134 & 12 & 2 & 0 & 3 & 171 \\
\hline $\mathrm{PE}$ & 19 & 133 & 9 & 2 & 0 & 5 & 168 \\
\hline $\mathrm{AL}$ & 24 & 56 & 9 & 4 & 1 & 3 & 97 \\
\hline $\mathrm{SE}$ & 14 & 33 & 19 & 8 & 0 & 0 & 74 \\
\hline BA & 30 & 186 & 59 & 38 & 16 & 86 & 415 \\
\hline Sudeste & 191 & 347 & 99 & 116 & 65 & 42 & 860 \\
\hline MG & 141 & 286 & 98 & 116 & 65 & 17 & 723 \\
\hline ES & 25 & 27 & 0 & 0 & 0 & 15 & 67 \\
\hline RJ & 25 & 34 & 1 & 0 & 0 & 10 & 70 \\
\hline Sul & 153 & 388 & 73 & 62 & 38 & 159 & 873 \\
\hline PR & 45 & 192 & 21 & 18 & 13 & 34 & 323 \\
\hline SC & 66 & 109 & 16 & 5 & 0 & 21 & 217 \\
\hline RS & 42 & 87 & 36 & 39 & 25 & 104 & 333 \\
\hline Centro-oeste & 15 & 116 & 55 & 50 & 6 & 136 & 378 \\
\hline MS & 6 & 40 & 2 & 1 & 0 & 23 & 72 \\
\hline MT & 3 & 29 & 1 & 0 & 0 & 62 & 95 \\
\hline GO & 6 & 47 & 52 & 49 & 6 & 51 & 211 \\
\hline Total Brasil & 696 & 1.834 & 372 & 304 & 126 & 586 & 3.918 \\
\hline
\end{tabular}




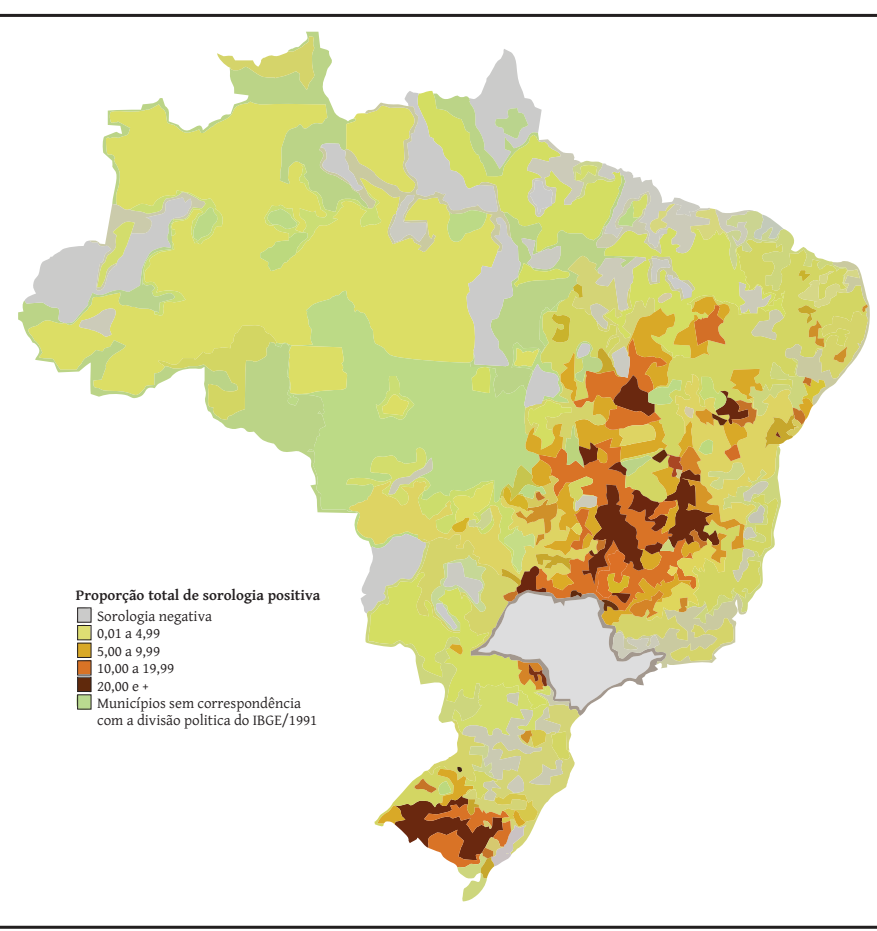

FIGURA 3 - Proporção de soropositivos por município. Inquérito sorológico nacional. Brasil. 1975-1980.

extraordinariamente altos, como na metade sul do Rio Grande do Sul e na região do Triângulo e norte de Minas Gerais, assim como no além São Francisco da Bahia e extremo norte do Paraná, todas essas áreas com infestações maciças por Triatoma infestans.

Os resultados do Inquérito sorológico da prevalência de infecção chagásica no Brasil,1975/1980, ao lado do Inquérito entomológico, que se realizou quase simultaneamente, foram absolutamente decisivos para a racionalização das atividades de controle da doença de Chagas no país.

\section{REFERÊNCIAS}

1. Camargo ME, Silva GR, Castilho EA, Silveira AC. Inquérito sorológico da prevalência de infecção chagásica no Brasil, 1975/1980. Rev Inst Med Trop São Paulo 1984; 26:192-204.

2. Villela E. A ocorrência de moléstia de Chagas em hospitais de Belo Horizontee na população de seu arredores. An Fac Med Univ Minas Gerais 1930; 1:1-18.

3. Pedreira de Freitas JL. Reação de fixação de complemento para diagnóstico da moléstia de Chagas pela técnica quantitativa [tese de doutorado]. [São Paulo]: Faculdade de Medicina da Universidade de São Paulo; 1951.

4. Dias E, Laranja F, Pellegrino J. Estudos sobre a importância social da doença de Chagas, 1: Inquérito clínico-epidemiológico feito nas vizinhanças de Bambuí, oeste de Minas. Brazil Med 1948; 62:412-413.
5. Freitas JLP, Figueiredo C. Resultados de investigações sorológicas sobre moléstia de Chagas realizadas no estado de Goiás. Arq Hig Saude Publica 1951; 16:227-230.

6. Brant TC, Laranja FS, Bustamante FM, Mello AL. Dados sorológicos e eletro-cardiográficos obtidos em populações não selecionadas de zonas endêmicas de doença de Chagas no Estado do Rio Grande do Sul. Rev Bras Malariol D Trop 1957; 9:141-148.

7. Martins AV. Parasitologia Médica. Rio de Janeiro: Guanabara Koogan; 1977.

8. Ponde A. A cardiopatia crônica na moléstia de Chagas. O Hospital 1960; 58:1074-1096.

9. Lucena DT. Estudos sobre doença de Chagas no Nordeste do Brasil. Rev Bras Malariol D Trop 1970; 22:3-173.

10. Porto CC, Porto C. Doença de Chagas no Triângulo Mineiro. Rev Goiana Med 1962; 8:21-34.

11. Salgado AA, Pellegrino J. Inquérito sorológico escolar sobre a doença de Chagas no Estado de Minas Gerais. Proceedings $7^{\text {th }}$ Int Congress Medic Mal 1963; 2:260.

12. Alencar JE, Almeida JO, Sherlock V, França AP, Leite L. Estudos sobre a epidemiologia da doença de Chagas no Ceará. II. Novos dados. Rev Bras Malariol D Trop 1963; 15:551-565.

13. Barros GC, Mayrink W, Abreu Salgado AA, Barros RCG, Sessa PA. Contribuição para o conhecimento da doença de Chagas autóctone no Estado do Espírito Santo. Rev Inst Med Trop São Paulo 1975; 17:319-329.

14. Coura JR, Rodrigues J. Um foco de doença de Chagas transmitida por Triatoma infestans na baixada fluminense, município de Duque de Caxias, estado do Rio de janeiro, Brasil. Rev Soc Bras Med Trop 1971; 5 : 123-128.

15. Salgado AA, Pellegrino J. Distribuição geográfica: Inquérito sorológico. In: Cançado JR, editors. Belo Horizonte: Imprensa Oficial do Estado de Minas Gerais; 1968. p.143-162.

16. Silveira AC, Sakamoto T, Faria Filho OF, Gil HSG. Sobre o foco de triatomíneos domiciliados na Baixada Fluminense. Rev Bras Malariol D Trop 1982; 34:50-58.

17. Camargo ME. Fluorescent antibody test for the serodiagnosis of Chagas'disease. Technical modification employing preserved cultural forms of Trypanosoma cruzi in a slid test. Rev Inst Med Trop São Paulo 1966; 8:227-234.

18. Castilho EA, Camargo ME, Silva GR, Silveira AC. Cálculo das estimativas de prevalência de infecção chagásica. Brasil 1975-1981. In: Resumos da I Reunião Anual de Pesquisa Aplicada sobre Doença de Chagas. Rev Soc Bras Med Trop 1984; 17 (supl):63.

19. Silveira AC, Feitosa VR, Borges R. Distribuição de triatomíneos capturados no ambiente domiciliar, no período de 1975-83, Brasil. Rev Bras Malariol D Trop 1984; 36:15-312.

20. Silveira AC, Passos ADC. Altos índices de prevalência sorológica de infecção chagásica em área da Amazônia. Rev Soc Bras Med Trop 1986; 19(supl): 45.

21. Coura JR, Barrett TV, Arboleda Naranjo M. Ataque de populações humanas por triatomíneos silvestres no Amazonas: uma nova forma de transmissão da infecção chagásica? Rev Soc Bras Med Trop 1994; 27:251-253.

22. Instituto Brasileiro de Geografia e Estatística. Censo Demográfico de 1980. Rio de Janeiro: IBGE; 1985.

23. Instituto Brasileiro de Geografia e Estatística. Censo Demográfico de 1991. Rio de Janeiro: IBGE; 1994. 